\title{
El S-commerce: La innovación a través de Medios Sociales
}

\author{
Alba Patricia Guzmán Duque
}

Resumen: La sociedad de la información ha favorecido la adopción del e-commerce como un mecanismo para ampliar los mercados, considerando las preferencias de las comunidades, donde los medios sociales son potentes herramientas que permiten el éxito en las transacciones a través del $s$ commerce. El estudio es descriptivo y se realizó a empresarios ( $\mathrm{N}=287$ ) del sector calzado en Bucaramanga (Santander-Colombia) para determinar su percepción sobre el uso de los medios sociales como estrategia de comercialización. Se destaca la utilización de Facebook, Instagram, YouTube y Twitter como los más aceptados por sus propiedades de conexión, y, Taobao, E-bay, Amazon, Linio y Mercado Libre, como canales oficiales de comercialización. Finalmente, se proponen estrategias para adoptar las TIC para incrementar la competitividad en las organizaciones.

Palabras clave: TIC; s-commerce; competitividad; sector calzado; medios sociales; e-commerce.

\begin{abstract}
The information s ociety has favored the a doption of e-commerce a s a mechanism to expand markets, considering the p references of communities, where social media are powerful tools that allow success in transactions through Of the s-commerce. The study is descriptive and was carried out to entrepreneurs $(\mathrm{N}=287)$ of the footwear sector in Bucaramanga (Santander-Colombia) to determine their perception about the use of social media as a marketing strategy. It highlights the use of Facebook, Instagram, YouTube and Twitter as the most accepted by its connection properties, and, Taobao, E-bay, Amazon, Linio and Mercado Libre, as official channels of marketing. Finally, strategies are proposed to adopt ICT to increase competitiveness in organizations.
\end{abstract}

Keywords: ICT; s-commerce; competitiveness; footwear sector; social media tools; e-commerce.

\section{Introduction}

El incremento de la tecnología ha permitido el desarrollo de la comunicación entre las organizaciones y las comunidades a través del uso de las Tecnologías de la Información y las Comunicaciones (TIC) favoreciendo el éxito en las transacciones (Guzmán, 2013). El e-commerce se ha convertido en un modelo a seguir para las organizaciones, considerando que la tendencia mundial obedece a la adopción de las TIC, como un elemento favorecedor de las dinámicas comunicativas y un mecanismo de innovación social (Maiolini, Marra, Baldassarri \& Carlei, 2016) que ha acercado a las organizaciones y a los consumidores (Webb, Gibson \& Forkosh, 2013).

Precisamente, los medios sociales de internet son una herramienta que favorece la interacción entre los usuarios, convirtiéndose en un mecanismo que permite a las organizaciones llegar de manera más directa a sus clientes debido a la confianza que tienen sobre la marca (Jiang, Tadikamalla, Shang \& Zhao, 2016). En este sentido, el s-commerce es una estrategia que crea un acercamiento entre el cliente final y la empresa disminuyendo los intermediarios a partir del uso de las TIC (Cui, Pan, Newell \& Cui, 2017). Sin embargo, los medios sociales son considerados como herramientas de ocio, dado que las redes sociales han facilitado la publicación de un sin número de información en ocasiones poco fundamentada, dada su fácil comunicación y manejo (Guzmán \& Del Moral, 2014).

Maiolini, Marra, Baldassarri y Carlei (2016) afirman que las pequeñas, medianas empresas y las start-up pueden utilizar las TIC para favorecer sus procesos de una manera innovadora, debido a que estas tecnologías trazan el futuro de las organizaciones en la globalización de las industrias.
Este artículo es producto de una investigación realizada en la Universidad Autónoma de Bucaramanga (Colombia) donde se buscó cómo aportar desde la academia a la mejora en los procesos comerciales del sector calzado con el uso de las herramientas sociales de internet para favorecer la apertura de nuevos mercados y el incremento de transacciones efectivas, detectándose el uso de los medios sociales -Facebook, Instagram, YouTube y Twitter- como canales informales de venta y los sitios -Taobao, E-bay, Amazon, Linio y Mercado Librecomo los canales formales establecidos para llegar al público objetivo.

\section{La Web Social y el e-commerce}

Es evidente que las TIC favorecen la comercialización de productos y servicios en una organización, donde el trabajo colaborativo dentro de la empresa y las transacciones entre las comunidades permite el incremento de las ventas en las organizaciones con la utilización de canales de comunicación basados en web social y diferentes plataformas, dispositivos móviles, marketing y e-commerce (Maiolini, Marra, Baldassarri \& Carlei, 2016). Esto se ha dado precisamente, porque los procesos de comunicación con el uso de internet han permitido su evolución para llegar al cliente final favoreciendo la competitividad de las organizaciones porque no hay intermediarios (Yan, Zheng, Wang, Song \& Zhang, 2015), debido a la facilidad que ofrece la web social para que los usuarios puedan expresar sus ideas creativas respaldadas por la comunidad permitiendo que la empresa adapte sus productos y/o servicios considerando sus preferencias (Fernandes \& Belo, 2016).

Diversos autores afirman que el e-commerce ofrece diferentes beneficios para las empresas. Erazo, Castro y Achicanoy (2016) aseguran que reduce los costos que se generan en los procesos organizacionales,

Programa Administración de Empresas modalidad virtual, Universidad Autónoma de Bucaramanga, Carrera 48, Calle 42 \# 40-11, Bucaramanga, Santander, Colombia, phone number 57 (7) 643 6111, aguzman201@unab.edu.co 
promoviendo la apertura de nuevos mercados y mejorando la atención de clientes nuevos y antiguos. Guzmán (2013) afirma que facilita el acceso de la empresa de una manera directa con los clientes favoreciendo la compra y evitando la presencialidad, incluyendo a las comunidades de todo el mundo. Hu, Huang, Zhong, Davison y Zhao (2016) indican que permite la optimización de los procesos, porque actúa en función del consumidor atendiendo sus deseos, casi personalizados y eliminando los intermediarios. Similar a como lo abordan Yapar, Bayrakdar y Yapar (2015) porque se facilita la comercialización casi mundial. Rahayu y Day (2015) confirman que las economías en vías de desarrollo pueden adoptar más fácil al e-commerce puesto que la inversión para su incursión es muy baja. Torres y Arroyo (2016) mencionan que su adopción surge como estrategia comercial para la mejora de la reputación de las organizaciones en el mercado, reduciendo de forma abrumadora la asimetría de la información e incrementando la aceptación de los productos o servicios.

Un aspecto importante a considerar en el e-commerce es la seguridad y la confiabilidad que requiere el usuario que compra por internet (Huang \& Benyoucef, 2017; Jiang, Tadikamalla, Shang \& Zhao, 2016). En este sentido, existen diferentes razones que han evitado la implementación del e-commerce en las organizaciones. Erazo, Castro y Achicanoy (2016) y Nilashi, Ibrahim, Mirabi, Ebrahimi y Zare (2015) indican que se trata de la desconfianza en la transacción y los costos que no se clarifican en el momento de la compra. Gomez, Martens y Turlea (2014) mencionan que los costos pueden verse incrementados porque la comunicación entre el consumidor y el vendedor no es clara, debido a diferencias lingüísticas, culturales, institucionales y comerciales. Savrul, Incekara y Sener (2014) precisan que la informalidad de quienes ofrecen el producto o servicio, influye en que el consumidor vea limitaciones relacionadas con la infraestructura y la seguridad para garantizar la transacción. Kurnia, Choudrie, Mahbubur y Alzougool (2015) aseguran que la implementación del e-commerce ha sido lenta porque en los países en vías de desarrollo, debido a las condiciones sociales, económicas, tecnológicas y políticas, aun cuando hay una mayor apropiación de este tipo de transacciones. Yapar, Bayrakdar y Yapar (2015) indican que la mayor falencia para adoptar esta estrategia es la incertidumbre en el tema tributario.

De otro lado, Torres y Arroyo (2016) afirman que el e-commerce ha superado las expectativas de rendimiento y de esfuerzo que esperaban los empresarios, así como la influencia social que la falta de claridad en el establecimiento de unas condiciones que faciliten su adopción, y el hábito de los consumidores hacia los precios bajos, la calidad, la confianza, la seguridad y la privacidad percibida, pese al riesgo que se considera se puede dar por la orientación hacia la innovación. Para contrarrestar esta problemática, Cui, Pan, Newell y Cui (2017) aseguran que se requiere esclarecer la naturaleza de la actividad que se desarrolla y el entorno de la organización, pues previamente las organizaciones que implementan el e-commerce requieren: gestionar sus capacidades, de acuerdo a sus recursos, gestionar su estructura y estrategias para fortalecer las capacidades que ya poseen y manejar los recursos para el desarrollo de las capacidades dinámicas que faciliten el éxito en la transacción.
El comercio a través de internet o e-commerce facilita la comercialización para las empresas a través de plataformas tecnológicas (Webb, Gibson \& Forkosh, 2013), diferenciándose con los canales tradicionales por la forma en que se desarrolla actualmente (Guzmán \& Del Moral, 2014). Permite la colaboración online entre los sujetos que intervienen en la transacción utilizando las TIC (Turban, Bolloju \& Liang, 2011), y proveen un entorno social que favorece la conversación participativa de los usuarios (Huang \& Benyoucef, 2017). De hecho, el e-commerce tiene tres elementos fundamentales: el uso de las tecnologías sociales, la interacción entre los usuarios y las comunidades y las actividades comerciales cerradas por una transacción (Turban, Bolloju \& Liang, 2011). Según el Top Sites Alexa (2017), los canales de venta de e-commerce más populares son Taobao, Amazon, E-bay, Olx, Mercadolibre, Tmail y Aliexpress.

Además, es necesario indicar que la evolución del e-commerce evidencia un avance hacia el s-commerce relacionando a las organizaciones de manera directa con los clientes finales disminuyendo los intermediarios y facilitando para el cliente la casi personalización de sus productos (Yan, Zheng, Wang, Song \& Zhang, 2015), utilizando como estrategia a los medios sociales, para avanzar hasta el s-commer$c e(\mathrm{Wu}$, Shen \& Chang, 2015). Las diferencias entre el e-commerce y el s-commerce radican principalmente en la forma como se comunican las empresas con sus consumidores y se orientan hacia la personalización (Huang \& Benyoucef, 2017), a ofrecer relaciones dinámicas (Yan, Zheng, Wang, Song \& Zhang, 2015), a la creación de contenidos conjuntos (Hajli \& Sims, 2015) y a la interacción entre las partes (Bai, Yao \& Dou, 2015) para cerrar una transacción (Wu, Shen \& Chang, 2015).

\section{De las redes sociales al s-commerce}

Los medios sociales en internet o herramientas que facilitan el $s$-commerce permiten la interacción entre los usuarios permitiendo el cierre de la transacción, aportando al desarrollo del comercio social (Bai, Yao \& Dou, 2015; Huang \& Benyoucef, 2017) a partir de las conversaciones online que favorecen la personalización de servicios para las comunidades (Huang \& Benyoucef, 2017), creando contenido colaborativo y agregando valor para el consumidor (Hajli \& Sims, 2015) siendo dinámicos y favoreciendo la reputación de la marca (Yan, Zheng, Wang, Song \& Zhang, 2015).

Existen diferentes definiciones de s-commerce. Wu, Shen y Chang (2015) indican que es el medio que combina a la tecnología de la web social con la satisfacción del consumidor evidenciada a través de una comunidad. Lin, Li y Wang (2017) aseguran que es una estrategia que permite a los empresarios, el manejo de sus negocios involucrando actividades comerciales relacionadas con el uso de herramientas de los medios sociales o de la web 2.0 que se orienta hacia los consumidores. Jacobsen y Barnes (2017) mencionan que es el sitio de intersección entre los sitios de comercio electrónico y las redes sociales para facilitar una transacción. Hwang y Kim (2016) afirman que es un subconjunto del e-commerce que utiliza las redes sociales para favorecer las interacciones con los usuarios. Ali y Busalim (2017) señalan que es una integración de las actividades que se generan alrededor de 
una transacción de manera social, donde los usuarios pueden difundir boca-a-boca sus experiencias de compra, conocimientos sobre los productos y proporcionar información sobre productos y servicios, a su red de contactos. Precisamente, Fernandes y Belo (2016) clasifican el uso de las redes sociales en: buscar conocimiento, interactuar con los clientes, lanzar nuevos productos, y, la comercialización de productos y servicios.

Un estudio realizado con las empresas de Algarve (Portugal) permitió determinar que las redes sociales ofrecen un potencial para la contratación de proyectos empresariales porque permiten acceder a un mayor número de opciones para los profesionales, interactuar con los clientes, lanzar nuevos productos y comercializarlos (Fernandes \& Belo, 2016).

De otro lado, la facilidad de las redes sociales como mecanismos de comunicación, ha facilitado la interacción entre las empresas y los usuarios, pues convocan usuarios con temas afines (Guzmán, 2013; Webb, Gibson \& Forkosh, 2013). Estos medios sociales son favorecedores de la innovación social porque permiten que las organizaciones se acerquen al consumidor de manera más directa. En una investigación realizada en China, se detectó que a través del e-commerce se facilitó la comercialización de los productos de los aldeanos del sector rural (Cui, Pan, Newell \& Cui, 2017), utilizando las herramientas del s-commerce.

Por otra parte, son diversos los propósitos de una estrategia basada en s-commerce. Pelc (2017) afirma que la interactividad que ofrecen las redes sociales permite la utilización de aplicaciones que favorezcan la difusión de las innovaciones de las organizaciones como una forma de mejorar los procesos comerciales. La Tabla 1 muestra algunos estudios realizados para precisar cuáles son las utilidades de los medios sociales para las organizaciones en el ámbito empresarial:

Tabla 1. Investigaciones realizadas para determinar la utilidad de los medios sociales a las empresas. Elaboración propia.

\begin{tabular}{|c|c|c|c|}
\hline Investigación realizada & Medios sociales & Propósito & Autores \\
\hline 519 universidades & YouTube & $\begin{array}{l}\text { Generar valor para la marca de la empresa y la necesidad } \\
\text { del community manager. }\end{array}$ & Guzmán \& Del Moral (2014) \\
\hline Estadísticas de popularidad & $\begin{array}{l}\text { Facebook, Twitter, YouTube, } \\
\text { Google+ y LinkedIn }\end{array}$ & $\begin{array}{l}\text { Promover nuevas fuentes de innovación para las } \\
\text { empresas. }\end{array}$ & Pelc (2017) \\
\hline 230 usuarios y 70 empresas & Facebook & $\begin{array}{l}\text { Favorecer la interacción con los clientes, y permitir el } \\
\text { lanzamiento de nuevos. }\end{array}$ & Fernandes \& Belo (2016) \\
\hline 421 usuarios & $\begin{array}{l}\text { Facebook, Twitter y } \\
\text { Pinterest }\end{array}$ & Determinar los motivos de compra de los consumidores. & Jacobsen \& Barnes (2017) \\
\hline 134 usuarios & $\begin{array}{l}\text { Facebook, LinkedIn y } \\
\text { Twitter }\end{array}$ & $\begin{array}{l}\text { Favorecer la confianza del consumidor por el respaldo de } \\
\text { la comunidad. }\end{array}$ & Lee, Cho \& Bae (2017) \\
\hline
\end{tabular}

Asimismo, las redes sociales han avanzado hasta su masificación considerando el incremento de su número de seguidores. De hecho, las herramientas sociales que hacen parte del s-commerce son: las redes sociales, los blogs, los foros, y las recomendaciones (Hajli \& Sims, 2015), los micro-blogs, las wikis, las plataformas de e-commerce y YouTube (Guzmán \& Del Moral, 2014), y, Flickr y las comunidades online (Bai, Yao \& Dou, 2015; Huang \& Benyoucef, 2017). La Tabla 2 evidencia el nivel de adopción de las primeras cinco herramientas de los medios sociales según el Top sites Alexa (2017) que mide el tráfico en las web de manera diaria -Facebook, YouTube, Wikipedia, Amazon y Twitter- donde el número de sitios vinculados se calcula con base en el número total de enlaces al sitio, el porcentaje de tráfico a través de las referencias que provienen de los motores de búsqueda, el tiempo en el sitio a partir del estimado diario en minutos y segundos por visitantes, y las páginas vistas con respecto a las páginas diarias únicas por visitante. 
Tabla 2. Distribución de las herramientas de medición del Top sites Alexa para determinar la popularidad de los medios sociales. Elaboración propia.

\begin{tabular}{|c|c|c|c|c|}
\hline Medio social & Sitios enlazados & \% de tráfico & Tiempo en el sitio & Páginas vistas \\
\hline Facebook & $7,601,185$ & $8.40 \%$ & $9: 48$ & 4.02 \\
\hline YouTube & $2,699,220$ & $15.50 \%$ & $8: 24$ & 4.94 \\
\hline Wikipedia & $1,722,890$ & $68.30 \%$ & $4: 11$ & 3.27 \\
\hline Amazon & 827,984 & $21.70 \%$ & $8: 05$ & 8.48 \\
\hline Twitter & $5,571,981$ & $12.10 \%$ & $6: 13$ & 3.27 \\
\hline
\end{tabular}

Se observa que Facebook tiene mayor número de sitios enlazados para facilitar el cierre de una transacción entre los usuarios, mientras que el mayor porcentaje de tráfico se encuentra en Wikipedia, el mayor número de páginas vistas en Amazon y el tiempo en el sitio es mayor en Facebook, YouTube y Amazon.

Lin, Li y Wang (2017) afirman que el uso de las herramientas del social media, interactuando con los sitios de e-commerce e incrementando su uso como canal de comercialización facilitan la adopción del s-commerce, donde Hwang y Kim (2016) aseveran que se requiere recrear un ambiente para la transacción confiable y basado en la seguridad que debe ofrecer la empresa a los clientes, y considerando los diferentes hábitos del cliente, mientras que Lee, Cho y Bae (2017) confirman que el cierre de la transacción impacta en el nivel de confianza que tiene el cliente con la empresa.

El s-commerce tiene tres orientaciones: a)hacia las organizaciones, considerando la reputación, la reputación de la empresa y el uso generalizado sobre contenido social; b) hacia la publicidad, a través de la investigación que se realiza en el tema, el uso del comercio móvil y la publicidad generada; c) hacia el boca-a-boca, revisiones de los medios sociales, interacción con los usuarios de la red, cultura, confianza generados y la adopción del marketing viral (Lin, Li \& Wang, 2017).

\section{Metodos}

\section{Participantes}

El estudio realizado es cuantitativo y buscó medir la percepción de 267 empresarios del subsector calzado de Bucaramanga (SantanderColombia) para determinar sus apreciaciones sobre el teletrabajo y la implementación del s-commerce como estrategia para la comercialización de sus productos y fue realizada en mayo de 2016. La antigüedad de estas empresas se encuentra "entre 3-5 años" el 28.2\%, "entre 5-10 años" el 25.4\%, "entre 1-3 años" el 20.2\%, "entre 10-20 años" el $13.2 \%$, "más de 20 años" el 7.0\% "menos de 1 año" el 5.9\%. De otro lado, el $64.4 \%$ afirmó que tiene solo una sede, el $24.3 \%$ que tiene 2, y el resto $11.3 \%$ tiene "entre 3-5 sedes". Las empresas tienen contratados “entre 1-10 empleados" el 72.5\%, "entre 10-20" el 18.1\%, "entre 20-40" el 5.9\%, y tan solo el 3.5\% tienen "más de 40 empleados". Entretanto, la formación de los empresarios se orienta hacia la educación media el 59.4\%, tecnologías el 19.6\%, universitarios el 14.3\%, nivel básico el $5.9 \%$, y tan solo el $0.3 \%$ tiene nivel de posgrado.

\section{Instrumento de medición}

El instrumento para la recolección de datos fue digital y se compuso por cinco secciones, cuatro de ellas relacionadas con este artículo: a) elementos descriptivos de la organización: edad, número de empleados, sedes, nivel educativo del empresario; b) percepción sobre la utilización del e-commerce: los productos que ofrece la empresa al público a través de imágenes; características, funcionalidades y/o precios de los productos para fomentar las ventas a través de modalidades tradicionales de venta; vender los productos a través de portales web de comercio electrónico; vender los productos a través de una plataforma electrónica desarrollada y gestionada por la organización; incrementar el número de clientes en el exterior para diversificar las ventas; conozco en qué consiste el e-commerce y entiendo los beneficios que puede aportar a la organización; el personal calificado es el apropiado para el desarrollo del e-commerce; es importante implementarlo, pero no se sabe por dónde iniciar; el conocimiento del e-commerce es básico; c) percepción sobre la utilización de los medios sociales: Facebook, YouTube, LinkedIn, Instagram, Twitter, Blog público, Blog de la empresa, Wikipedia; $y$, d) percepción sobre la utilización de los canales de venta de e-commerce: Amazon; Mercado Libre; Linio; Olx; Ebay; Taobao; e) aspectos relacionados con el teletrabajo y las competencias del teletrabajador, no abordados en esta investigación.

La fiabilidad del instrumento se midió a partir de la respuesta previa de diez empresarios quienes no están incluidos dentro de los resultados y entregando un alfa de Cronbach=.902, evidenciando que es fiable para continuar con la investigación (Hair, Prentice, Cano \& Suárez, 2007).

\section{Diseño del estudio}

Las variables estudiadas son de tipo cualitativo y las preguntas se diseñaron bajo la escala de Likert de 1 a 5 (1=Totalmente en desacuerdo, 2=En desacuerdo; 3=Neutro; 4=De acuerdo; $5=$ Totalmente de acuerdo). Todas las variables son independientes puesto que el propósito de la investigación no es crear un modelo, sino describir la percepción que tienen las empresas comercializadoras de calzado a través de herramientas sociales de internet y proponer estrategias para que se mejore este proceso en el sector.

\section{Procedimiento}

Se utilizaron técnicas estadísticas de carácter descriptivo para la contextualización de la muestra, la técnica del ANOVA para determinar 
la influencia de las variables edad de las empresas y formación académica de los empresarios en las otras varibles, y como técnica multivariante se utilizó el análisis factorial con el método componentes principales y la variación varimax para recargar los pesos de las variables en los componentes (Hair, et al., 2007), para precisar los factores que influyen en la aceptación del s-commerce como mecanismo para la comercialización de los productos. El paquete estadístico utilizado fue SPSS versión 23.

\section{Resultados}

\section{La utilización del e-commerce}

El 49.2\% de los empresarios afirma que desde el 2015 utilizan el $e$ commerce como canal de comercialización, donde el 30.1\% no tiene una estrategia planteada que se relacione con este aspecto, como ventajas, el $84.1 \%$ indica que favorece la creación de lazos comerciales $y$ nuevos mercados, el $76.1 \%$ que es un proceso innovador, el $67.3 \%$ que aporta a la negociación entre las partes, el $61.1 \%$ que permite la contratación laboral, el 54.9\% que mejora el servicio al cliente y el $39.0 \%$ que aporta a la disminución de costos y gastos.

La Figura 1 evidencia la opinión de los empresarios con respecto a los objetivos que se pueden lograr con la utilización del e-commerce, destacándose en el nivel totalmente de acuerdo: los productos que ofrece la empresa al público a través de imágenes (36.3\%) y las características y funcionalidades que fomentan en las ventas a través de los canales tradicionales (29.1\%); similar ocurre en el nivel de acuerdo donde estos ítem representan el $39.7 \%$ y el $37.0 \%$, respectivamente, seguidos por la venta de productos a través de una plataforma electrónica propia (33.9\%) y el incremento del número de clientes en el exterior para la diversificación de las ventas (30.5\%). Es importante destacar que estos dos últimos ítem tienen una representación del $28.1 \%$ para los empresarios, en cuanto a que están totalmente en desacuerdo con estas apreciaciones.

Figura 1. Distribución porcentual de la opinión de los empresarios con respecto a los objetivos que se pueden lograr con la utilización del e-commerce. Elaboración propia.

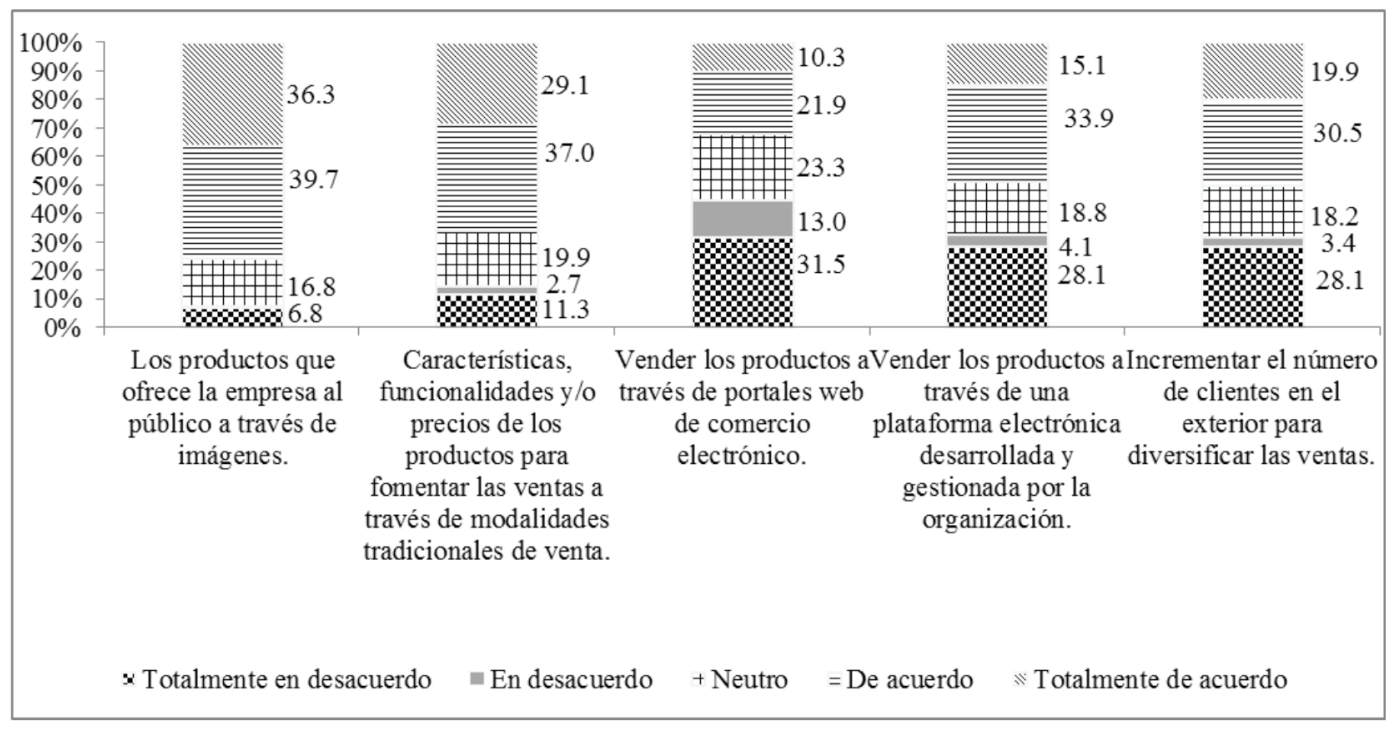

De manera similar a la distribución anterior, la Figura 2 evidencia el grado de acuerdo de los empresarios en cuanto a las razones por las cuales no se ha implementado el e-commerce como estrategia comercial dentro de sus organizaciones, donde los empresarios siguen considerando en los niveles totalmente de acuerdo y de acuerdo las diferentes razones, destacándose en el nivel totalmente de acuerdo, el conocimiento del e-commerce es básico (25.0\%), seguido por el conocimiento sobre en qué consiste y los beneficios que trae para la empresa (22.9\%). Es de destacar que en el nivel totalmente en desacuerdo los ítems es importante implementarlo pero no se sabe cómo iniciar (30.5\%) es el más destacado. 
Figura 2. Distribución porcentual del grado de acuerdo de los empresarios en cuanto a las razones por las cuales no se ha implementado el e-commerce. Elaboración propia.

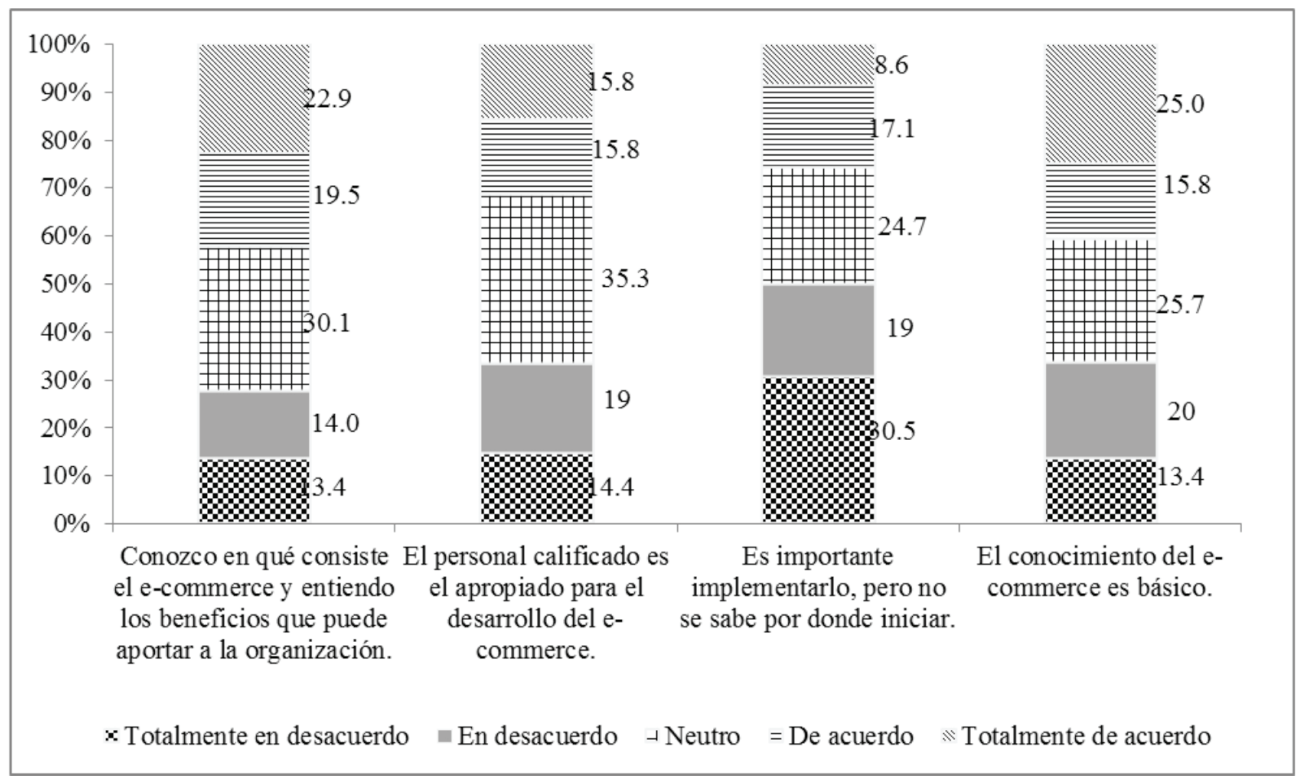

Al realizar el análisis del ANOVA con la variable edad, se determina que las empresas presentan diferencias significativas al considerar la realización de reuniones de trabajo ( $\mathrm{p}<0.001)$, además con la percepción de la utilidad que tiene el e-commerce para el desarrollo de las actividades comerciales ( $\mathrm{p}<0.003)$, con el objetivo de la organización vender los productos a través de una plataforma electrónica desarrollada y gestionada por la organización ( $\mathrm{p}<0.000)$, y, en las razones que se tienen presentes para adoptar el e-commerce porque se conoce en qué consiste el e-commerce y se entienden los beneficios que puede aportar a la organización ( $\mathrm{p}<0.054$ ), donde son las organizaciones más antiguas las que ven la importancia de las anteriores apreciaciones sobre el e-commerce.

Con el análisis del ANOVA considerando la variable formación del empleador, se determina que las empresas presentan diferencias significativas en cuanto a los objetivos de la organización vender los productos a través de una plataforma electrónica desarrollada y gestionada por la organización ( $\mathrm{p}<0.006)$, en la importancia para dar a conocer las características de los productos de la empresa para fomentar las ventas a través de internet $(\mathrm{p}<0.014)$, con las razones que se tienen presentes para adoptar el e-commerce porque se conoce en qué consiste el e-commerce y se entienden los beneficios para la organización $(\mathrm{p}<0.000)$, el personal calificado es el apropiado para el desarrollo del e-commerce $(\mathrm{p}<0.000)$, es importante implementarlo, pero no se sabe por dónde iniciar $(\mathrm{p}<0.001)$ y el conocimiento del e-commerce es básico $(\mathrm{p}<0.000)$, donde las organizaciones más jóvenes destacan esta importancia.

Lo anterior se ve reflejado en la utilización de los canales más populares para la comercialización, donde los empresarios destacan como los más utilizados Taobao (98.6\%), E-bay (97.9\%), Amazon (97.6\%), Linio (97.3\%) y Mercado Libre (93.2\%).

\section{La adopción del s-commerce}

La Tabla 3 evidencia el grado de percepción de las ventajas que ofrecen las herramientas de los medios sociales para el desarrollo de la actividad económica de la empresa. Se destacan como las más utilizadas en la comercialización de los productos en los niveles Totalmente de acuerdo $y$ de acuerdo Twitter (79.8\%), YouTube (77.4\%), Facebook (66.4\%) e Instagram (42.8\%), y como las menos utilizadas en los niveles en desacuerdo y totalmente en desacuerdo Wikipedia (88.0\%) y LinkedIn (87.0\%).

Tabla 3. Distribución porcentual de la percepción de las ventajas que ofrecen los medios sociales considerando sus ventajas. Elaboración propia.

\begin{tabular}{llllll}
\hline Medio social & Totalmente en desacuerdo & En desacuerdo & Neutro & De acuerdo & Totalmente de acuerdo \\
\hline Facebook & 3.4 & 16.8 & 13.4 & 26.0 & 40.4 \\
YouTube & 7.5 & 4.8 & 10.3 & 11.6 & 65.8 \\
LinkedIn & 74.7 & 12.3 & 4.1 & 4.5 & 4.5 \\
Instagram & 7.9 & 31.8 & 17.8 & 16.1 & 26.4 \\
Twitter & 7.5 & 5.1 & 7.5 & 12.3 & 67.5 \\
Blog público & 56.2 & 12.0 & 10.3 & 11.6 & 9.9 \\
Blog de la empresa & 50.3 & 13.7 & 6.8 & 9.2 & 12.0 \\
Wikipedia & 78.1 & 2.4 & 13.4 & 9.9 & 2.7 \\
Baidu & 40.4 & 26.0 & 3.4 & 16.8 \\
\hline
\end{tabular}

ISSN: 0718-2724. (http://jotmi.org) 
Entretanto, la Tabla 4 muestra la distribución de la percepción que tienen los empresarios en cuanto a la utilización de las herramientas de los medios sociales como canales de venta a través de internet, donde en los niveles de acuerdo y totalmente de acuerdo se destacan Facebook
(89.3\%), YouTube (80.5\%), Twitter (77.4\%) e Instagram (54.5\%), mientras que en los niveles totalmente en desacuerdo y en desacuerdo se encuentran como los menos utilizados Baidu (94.9\%), Wikipedia (93.8\%), LinkedIn (92.5\%) y los blogs públicos (78.1\%) y de la empresa(71.9\%).

Tabla 4. Distribución porcentual de grado de acuerdo en cuanto a la utilización de los medios sociales en las empresas. Elaboración propia.

\begin{tabular}{llllll}
\hline Medio social & Totalmente en desacuerdo & En desacuerdo & Neutro & De acuerdo & Totalmente de acuerdo \\
\hline Facebook & 4.0 & 5.1 & 1.6 & 33.7 & 55.6 \\
YouTube & 3.8 & 9.2 & 6.5 & 1.4 & 79.1 \\
LinkedIn & 83.6 & 8.9 & 4.5 & 1.0 & 2.1 \\
Instagram & 24.0 & 11.3 & 10.3 & 4.8 & 49.7 \\
Twitter & 7.2 & 9.9 & 5.5 & 1.0 & 76.4 \\
Blog público & 69.9 & 8.2 & 11.3 & 6.8 & 6.5 \\
Blog de la empresa & 63.4 & 8.6 & 5.1 & 6.2 & 10.6 \\
Wikipedia & 87.3 & 6.5 & 4.8 & 0 & 1.0 \\
Baidu & 88.7 & 6.2 & & 0 & .3 \\
\hline
\end{tabular}

Al realizar el análisis del ANOVA con la variable edad, se determina que las empresas presentan diferencias significativas destacándose el uso de Facebook ( $\mathrm{p}<0.007)$, YouTube $(\mathrm{p}<0.000)$ y Twitter $(\mathrm{p}<0.000)$. De manera similar ocurre con estos medios sociales y la percepción sobre las ventajas que les ofrecen estos sitios a los empresarios ( $\mathrm{p}<$ $0.051 ; \mathrm{p}<0.001 ; \mathrm{p}<0.000)$, donde son las organizaciones más antiguas quienes destacan la importancia de las anteriores apreciaciones sobre el s-commerce. Es necesario destacar que no existen diferencias significativas considerando la edad de las empresas y la utilidad que ofrecen como canal de venta online.

Con el análisis del ANOVA y la variable nivel educativo se determina que las empresas presentan diferencias significativas en cuanto al uso de Facebook ( $\mathrm{p}<0.053)$, YouTube ( $\mathrm{p}<0.045)$, LinkedIn (0.000), Instagram ( $\mathrm{p}<0.000)$, Twitter $(\mathrm{p}<0.000)$ y Wikipedia $(0.036)$. De manera similar en cuanto a las ventajas que les ofrecen, estos sitios $(\mathrm{p}<0.054$; $\mathrm{p}<0.045 ; \mathrm{p}<0.000 ; \mathrm{p}<0.000 ; \mathrm{p}<0.000 ; \mathrm{p}<0.000$ ), donde son las organizaciones más antiguas las que ven la importancia de las anteriores apreciaciones sobre el s-commerce. En cuanto a la percepción sobre la utilidad que ofrecen los canales online de distribución de los productos se destaca que todos son significativos.

\section{La innovación a través del s-commerce}

Con la utilización de la técnica análisis factorial se detectaron las razones que los empresarios valoran como importantes para la implementación del e-commerce. Un primer componente se orienta hacia dos razones: de conocimiento, compuesto por el conocimiento de la estrategia y sobre los beneficios que puede aportar, la calidad de los profesionales para desempeñarse en el área, y de experiencia, relacionada con que se es consciente de la importancia de implementarlo, pero no se sabe por dónde iniciar $(\mathrm{KMO}=0.721$; Prueba de esfericidad de Bartlett Chi-cuadrado=439.295 con gl=6 y p $<0.000$; VE=84.846).

Por otra parte, en cuanto a los objetivos de las organizaciones para la utilización del s-commerce, son dos los componentes principales: promoción relacionado con la necesidad de dar a conocer los productos a través de imágenes y dar a conocer sus características y funcionalidades para incrementar las ventas; uso considerando la venta a través de la plataforma electrónica de la organización y el incremento de los clientes en el exterior para incrementar las ventas $(\mathrm{KMO}=0.547$; Prueba de esfericidad de Bartlett Chi-cuadrado $=366.514$ con gl $=6$ y $\mathrm{p}<0.000 ; \mathrm{VE}=83.209$ ).

De otro lado, el uso de los medios sociales como mecanismos para promocionar los productos de las empresas se orienta hacia dos clases: generales orientadas a todo tipo de público, donde hacen parte Facebook, Twitter e Instagram; y especializadas donde se encuentran YouTube, Wikipedia y LinkedIn que no tienen como motivo realizar campañas de comercialización de manera directa $(\mathrm{KMO}=0.684$; Prueba de esfericidad de Bartlett Chi-cuadrado=827.426 con $\mathrm{gl}=21$ y $\mathrm{p}<0.000$; VE=80.412). Similar agrupación por componente se encuentra en relación a las ventajas que son reconocidas por las organizaciones (KMO=0.814; Prueba de esfericidad de Bartlett Chi-cuadrado=1070.038 con $\mathrm{gl}=15$ y $\mathrm{p}<0.000 ; \mathrm{VE}=80.153$ ).

Finalmente, en relación al uso de canales específicos para la distribución de productos online, se encuentran en un solo componente todos los canales: Amazon, Mercado Libre, Linio, Olx, Ebay y Taobao (KMO=0.893; Prueba de esfericidad de Bartlett Chi-cuadra$\mathrm{do}=2478.794$ con $\mathrm{gl}=15$ y $\mathrm{p}<0.000 ; \mathrm{VE}=80.510$ ).

\section{Discusión}

\section{Diagnóstico del sector calzado}

Es evidente el desconocimiento de los empresarios del sector para utilizar los canales de comercialización virtuales, por la desconfianza que aún perciben relacionada con la parte lúdica que ofrece internet. Sin embargo, se destaca la importancia que ven en la búsqueda de nuevos mercados que les permitan el incremento de sus transacciones por fuera de la región a que pertenecen. Esto implica que los empre- 
sarios reconocen los beneficios del e-commerce y del s-commerce pero los aspectos relacionados con la falta de confianza y el conocimiento sobre su funcionamiento e implementación impiden que esta estrategia tenga un mayor éxito.

Los objetivos de los empresarios con respecto a la utilización del $e$ commerce presentan una opinión dispar, debido a que la mayoría ven la importancia que tienen los canales, pero aún no están convencidos sobre el aporte que hacen como mecanismos de comercialización para sus productos y servicios, precisamente porque se confunden con el carácter lúdico que ofrecen las redes sociales.

La principal razón que incide en que los empresarios no hayan iniciado sus planes de e-commerce en sus empresas se relaciona con el desconocimiento del proceso, y porque no se ha establecido un plan para desarrollar esta estrategia comercial, por este motivo continúan con la utilización de los canales tradicionales de venta, pese a la importancia que han determinado sobre las transacciones comerciales y en la búsqueda de nuevos mercados. Esto se refuerza porque la fuerza productiva que ellos poseen no se encuentra capacitada para desarrollar actividades comerciales por internet y toda la parte estratégica recae en los empresarios, viendo la necesidad de contratar personal que se encargue solo de manejar sus canales de venta online y una posibilidad para esto es la contratación a través de teletrabajo.

Es un hecho que Facebook es la red social más utilizada como canal de venta online informal, puesto que su popularidad favorece la llegada a mayor número de usuarios de la red y su voz-a-voz favorece la interacción entre los usuarios que ofrece el respaldo de las comunidades virtuales. De otro lado, canales especializados como mercado Libre, Amazon, Linio, Oxl son los preferidos por los empresarios como favorecedores para la venta online, sin embargo, dado el desconocimiento de los empresarios por el manejo de estos portales las transacciones virtuales no ocupan un lugar significativo dentro de sus administraciones, pero están abiertos a buscar alternativas para comercializar sus productos a través de estos medios precisamente para abrir sus canales de venta.

Es importante destacar que los empresarios consideran como importantes para la comercialización a los medios sociales, debido a que favorecen la venta de los productos y aminora los costos y gastos, $\mathrm{y}$ además se incrementan los clientes a través de la promoción online barata y el voz-a-voz que ofrecen los medios sociales. Precisamente, los empresarios destacan que sus canales de venta oficiales son Taobao, E-bay, Amazon, Linio y Mercado Libre, mientras que como canales informales de venta se encuentran Facebook, Twitter, YouTube e Instagram.

\section{Prácticas innovadoras para el sector}

Es necesario que los empresarios consideren crear y desarrollar sus canales de comercialización online de manera que se efectúe un incremento en sus ventas hacia sectores dentro y fuera de la región, pero que se tenga presente el mecanismo que se utilizará para los despachos a domicilio de los productos, pues este puede ser un factor que impide la competitividad del sector.
Es necesario que se tengan presente dos objetivos para la utilización del s-commerce, y que se orientan hacia la promoción para dar a conocer los productos, características y funcionalidades para el incremento de las ventas y el uso de los canales de venta a través de la web de la empresa y del uso de los medios sociales para incrementar el número de clientes que compren los productos. De esta manera será más eficiente el proceso de comercialización de los productos y servicios de la empresa.

Es necesario que los empresarios organicen sus estrategias de venta en s-commerce considerando el tipo de medio social hacia donde se dirigen los esfuerzos de comercialización dado el reconocimiento por su popularidad y base de datos de clientes para la comercialización de los productos y que se orientan hacia: el uso de medios sociales generales como Facebook, Twitter e Instagram, y la utilización de medios sociales especializados como YouTube, Wikipedia y LinkedIn.

La contratación laboral es un elemento esencial que se requiere implementar, debido a que la fuerza laboral que tienen las empresas de calzado de la región se orienta principalmente a la fabricación de calzado y no tienen ni el conocimiento ni la experiencia para encargarse del canal de venta online de la empresa y mucho menos del manejo a través del voz-a-voz, pues el conocimiento sobre el tema se limita a sus operaciones personales. Por este motivo, las empresas deben buscar la contratación a través de teletrabajo permitiendo que este canal tenga una atención más centrada en el cliente de internet.

Por otra parte, es necesario que en el sector se consideren dos tipos de estrategias para la implementación del e-commerce, por un lado, se requiere la realización de talleres o cursos que faciliten incrementar el conocimiento de los empresarios con respecto a la estrategia, considerando que son conscientes de la importancia de este mecanismo, y por el otro ofrecer casos de éxito de pequeños empresarios que ya hayan implementado el s-commerce como alternativa de comercialización para facilitar la confianza en la contratación de personal para el manejo del canal de venta online.

\section{References}

Alexa. (2017). Top sites Alexa. Recuperado de https://www.alexa. com/topsites

Ali, S. Y., \& Busalim, A. H. (2017, July). Antecedents of eWOM in social commerce. In Research and Innovation in Information Systems (ICRIIS), 2017 International Conference on (pp. 1-6). IEEE. doi:10.1109 / ICRIIS.2017.8002482

Bai, Y., Yao, Z., \& Dou, Y. F. (2015). Effect of social commerce factors on user purchase behavior: An empirical investigation from renren. com. International Journal of Information Management, 35(5), 538550. doi:10.1016/j.ijinfomgt.2015.04.011

Cui, M., Pan, S. L., Newell, S., \& Cui, L. (2017). Strategy, Resource Orchestration and E-commerce Enabled Social Innovation in $\mathrm{Ru}-$ ral China. The Journal of Strategic Information Systems, 26(1), 3-21. doi:10.1016/j.jsis.2016.10.001 
Erazo, S., C. R., Castro, A. A., \& Achicanoy, H. A. (2016). Inversión en Tecnologías de la Información y las Comunicaciones y su relación con en el direccionamiento estratégico de las PYMES de Santiago de Cali-Colombia. RISTI-Revista Ibérica de Sistemas e Tecnologias de Informação, (18), 01-17. doi:10.17013/risti.18.1-17

Fernandes, S., \& Belo, A. (2016). Social networks as enablers of enterprise creativity: evidence from portuguese firms and users. Journal of technology management \& innovation, 11(2), 76-85. doi: 10.4067/ S0718-27242016000200008

Gomez, H., E., Martens, B., \& Turlea, G. (2014). The drivers and impediments for cross-border e-commerce in the EU. Information Economics and Policy, 28, 83-96. doi:10.1016/j.infoecopol.2014.05.002

Guzman, A. P. (2013). Factores críticos en el uso de las redes sociales: una aplicación a Twitter. (Doctoral dissertation, Editorial Universitat Politècnica de València).

Guzmán, A. P., \& Moral, M. E. D. (2014). Tendencias de uso de YouTube: optimizando la comunicación estratégica de las universidades iberoamericanas. Observatorio $\left(O B S^{*}\right), 8(1), 69-94$.

Hajli, N., \& Sims, J. (2015). Social commerce: The transfer of power from sellers to buyers. Technological Forecasting and Social Change, 94, 350-358. doi:10.1016/j.techfore.2015.01.012

Huang, Z., \& Benyoucef, M. (2017). The effects of social commerce design on consumer purchase decision-making: an empirical study. Electronic Commerce Research and Applications, 25, 40-58. doi:10.1016/j.elerap.2017.08.003

Hwang, I. H., \& Kim, J. S. (2016). A Study on the Effect of Social Commerce's Trust-Building on Intention of Continuous Use. Journal of Information Technology Applications and Management, 23(1), 1-23. doi: 10.21219 / jitam.2016.23.1.001

Hu, X., Huang, Q., Zhong, X., Davison, R. M., \& Zhao, D. (2016). The influence of peer characteristics and technical features of a social shopping website on a consumer's purchase intention. International Journal of Information Management, 36(6), 1218-1230. doi:10.1016/j. ijinfomgt.2016.08.005

Jacobsen, S., \& Barnes, N. G. (2017). On Being Social: How Social Identity Impacts Social Commerce for the Millennial Shopper. International Journal of Management Science and Business Administration, 3(2), 38-45. doi:10.18775/ijmsba.1849-5664-5419.2014.34.1005

Jiang, G., Tadikamalla, P. R., Shang, J., \& Zhao, L. (2016). Impacts of knowledge on online brand success: an agent-based model for online market share enhancement. European Journal of Operational Research, 248(3), 1093-1103. doi:10.1016/j.ejor.2015.07.051

Kurnia, S., Choudrie, J., Mahbubur, R. M., \& Alzougool, B. (2015). E-commerce technology adoption: A Malaysian grocery SME retail sector study. Journal of Business Research, 68(9), 1906-1918. doi:10.1016/j.jbusres.2014.12.010
Lee, C. C., Cho, Y. S., \& Bae, B. B. (2017). Factors affecting trust in social comerce: a structural equation model. Issues in Information Systems, 18(4), 70-90.

Lin, X., Li, Y., \& Wang, X. (2017). Social commerce research: Definition, research themes and the trends. International Journal of Information Management, 37(3), 190-201. doi:10.1016/j.ijinfomgt.2016.06.006

Maiolini, R., Marra, A., Baldassarri, C., \& Carlei, V. (2016). Digital Technologies for Social Innovation: An Empirical Recognition on the New Enablers. Journal of technology management \& innovation, 11(4), 22-28. doi:10.4067/S0718-27242016000400004

Nilashi, M., Ibrahim, O., Mirabi, V. R., Ebrahimi, L., \& Zare, M. (2015). The role of Security, Design and Content factors on customer trust in mobile commerce. Journal of Retailing and Consumer Services, 26, 57-69. doi:10.1016/j.jretconser.2015.05.002

Pelc, K. I. (2017). Diffusion of Innovation in Social Networking. In Technology, Society and Sustainability (pp. 3-13). Springer International Publishing.

Rahayu, R., \& Day, J. (2015). Determinant factors of e-commerce adoption by SMEs in developing country: evidence from Indonesia. Procedia-Social and Behavioral Sciences, 195, 142-150. doi:10.1016/j. sbspro.2015.06.423

Savrul, M., Incekara, A., \& Sener, S. (2014). The potential of e-commerce for SMEs in a globalizing business environment. Procedia-Social and Behavioral Sciences, 150, 35-45. doi:10.1016/j.sbspro.2014.09.005

Torres, J., A. S., \& Arroyo, C., F. J. (2016). Diferencias de la adopción del comercio electrónico entre países. Suma de Negocios, 7(16), 141150. doi:10.1016/j.sumneg.2016.02.008

Turban, E., Bolloju, N., \& Liang, T. P. (2011). Enterprise social networking: Opportunities, adoption, and risk mitigation. Journal of $\mathrm{Or}$ ganizational Computing and Electronic Commerce, 21(3), 202-220. doi: 10.1080/10919392.2011.590109

Webb, M., Gibson, D., \& Forkosh, A. (2013). Challenges for information technology supporting educational assessment. Journal of Computer Assisted Learning, 29(5), 451-462. doi:10.1111/jcal.12033

Wu, Y., C. J., Shen, J. P., \& Chang, C. L. (2015). Electronic service quality of Facebook social commerce and collaborative learning. Computers in human behavior, 51, 1395-1402. doi:10.1016/j.chb.2014.10.001

Yan, S. R., Zheng, X. L., Wang, Y., Song, W. W., \& Zhang, W. Y. (2015). A graph-based comprehensive reputation model: Exploiting the social context of opinions to enhance trust in social commerce. Information Sciences, 318, 51-72. doi:10.1016/j.ins.2014.09.036

Yapar, B. K., Bayrakdar, S., \& Yapar, M. (2015). The Role of Taxation Problems on the Development of E-Commerce. Procedia-Social and Behavioral Sciences, 195, 642-648. doi:10.1016/j.sbspro.2015.06.145 
